\title{
Venus Lower Atmosphere Heat Balance
}

\author{
Andrew P. Ingersoll and Judith B. Pechmann
}

Division of Geological and Planetary Sciences, California Institute of Technology, Pasadena, California 91125

\begin{abstract}
Pioneer Venus observations of temperatures and radiative fluxes are examined in an attempt to understand the thermal balance of the lower atmosphere. If all observations are correct and the probe sites are typical of the planet, the second law of thermodynamics requires that the bulk of the lower atmosphere heating must come from a source other than direct sunlight or a thermally driven atmospheric circulation. Neither the so-called greenhouse models nor the mechanical heating models are consistent with this interpretation of the observations. One possible interpretation is that two out of the three probe sites are atypical of the planet. Additional lower atmosphere heat sources provide another possible interpretation. These include a planetary heat flux that is $\mathbf{2 5 0}$ times the earth's, a secular cooling of the atmosphere, and a chemically energetic rain carrying solar energy from the clouds to the surface. Other data make these interpretations seem unlikely, so measurement error remains a serious possibility.
\end{abstract}

\section{INTRODUCTION}

Understanding the thermal balance of Venus, particularly the high surface temperatures, is one of the major objectives of the Pioneer Venus mission [Schubert et al., 1977, this issue; Tomasko et al., 1977, this issue (a)]. Measurements of temperatures and pressure [Sieff et al., 1979, this issue; Taylor, et al., 1979, this issue], net solar flux [Tomasko et al., 1979, this issue (b)], and net infrared flux [Boese et al., 1979, this issue; Suomi et al., 1979] were expected to satisfy this basic objective. It is shown below that barring measurement error and assuming the probe sites are typical of the planet, the measurements cannot satisfy the second law of thermodynamics unless additional heat sources are present.

Before Pioneer Venus the so-called greenhouse effect seemed to be the process most likely responsible for the high surface temperatures [Sagan, 1960]. Downward transport of mechanical energy had also been proposed [Goody and Robinson, 1966; Schubert et al., 1977]. Since the ultimate energy source in both models is thermal, the sources must be consistent with the second law. To satisfy this constraint, the atmosphere must emit infrared radiation at a lower average temperature than that at which sunlight is absorbed. Our analysis of the Pioneer Venus observations which takes into account the vertical and horizontal distribution of heat sources implies that the opposite is true.

To avoid a contradiction, one must assume either that some of the data are in error, that the probe locations are atypical of the planet, that the lower atmosphere and/or the planet are losing net heat, or that the solar energy absorbed at high altitudes somehow reaches the surface as a chemically energetic rain. These possibilities are discussed in section 3 . Section 2 shows how the data are used to test for thermodynamic consistency.

\section{METHOD}

Consider the entire atmosphere as a thermodynamic system with entropy $S$. In a statistically steady state the time rate of change $S$ must be zero. Thus the second law of thermodynamics requires

$$
\int Q d m / T \leq S=0 .
$$

Here $Q d m$ is the rate of heat transfer to an atmospheric mass element $d m$ from sources outside the system, e.g., the sun, the

Copyright $(\mathcal{C} 1980$ by the American Geophysical Union. solid planet, and space. $T$ is the temperature of the mass element; the temperature of the source is irrelevant to this argument. For example, when sunlight is absorbed, the relevant temperature is that at which a change of atmospheric internal energy occurs.

If the atmosphere and solid planet are in a statistically steady state, there can be no net work done and no net heat transfer to the environment. (We are, for the present, neglecting tidal work and internal heating in comparison to absorbed sunlight.) Thus for a suitably long time average,

$$
\int Q d m=0
$$

Equations (1) and (2) imply that the heat inputs to the atmosphere $(Q>0)$ must be at a higher temperature, on average, than the heat outputs $(Q<0)$.

In adapting the above expressions to Pioneer Venus data it is convenient to divide by the area of the planet and use an overbar to denote the areal average. We use pressure as a vertical coordinate and treat the surface pressure $P_{0}$ as constant. Thus we define

$$
\begin{gathered}
Q d m=-\frac{\partial}{\partial p}\left(F_{s}-F_{1}-F_{c}\right) d P d_{\text {Area }} \\
\bar{F}_{s}=F_{i} \quad \text { at } \quad P=0 \\
F_{c}=\bar{F}_{s}-\bar{F}_{i} \quad \text { at } \quad P=P_{0}
\end{gathered}
$$

Here $F_{s}$ is the net solar flux (power per unit area) downward, $F$, is the net infrared flux upward, and $F_{\mathrm{c}}$ is the heat flux conducted upward from the surface to the atmosphere. The latter is assumed to fall rapidly to zero above the surface. Equations (3)-(5) automatically satisfy (2). $F_{c}$ may be eliminated by the further assumption

$$
F_{c}=F_{s}=F_{i}=0 \quad \text { at } \quad P=P_{0}
$$

which is equivalent to having energy at the surface exchanged immediately with the lower atmosphere. Use of (6) makes our estimate of $\int Q d m / T$ more negative, since sunlight reaching the ground is then transferred to the atmosphere at its warmest point.

Pioneer Venus observations suggest that the largest hori- 
TABLE 1a. Profiles for the Standard Model

\begin{tabular}{rllrc}
\hline$Z, \mathrm{~km}$ & $P$, bar & $T, \mathrm{~K}$ & $\Delta T, \mathrm{~K}$ & $\boldsymbol{F}_{s}, \mathbf{W ~ m}^{-2}$ \\
\hline 0 & 91.0 & 732.8 & -8.6 & $\mathbf{0}$ \\
5 & 65.3 & 693.9 & -7.0 & 15 \\
10 & 47.0 & 655.2 & -10.7 & 15 \\
15 & 32.1 & 614.9 & -2.5 & 16 \\
20 & 21.8 & 574.1 & 0.8 & 20 \\
25 & 14.3 & 532.4 & 2.1 & 24 \\
30 & 9.09 & 490.5 & 0.8 & 27 \\
35 & 5.55 & 449.9 & 0.6 & 30 \\
40 & 3.26 & 412.7 & 3.5 & 32 \\
45 & 1.83 & 381.8 & 7.0 & 33 \\
50 & 0.984 & 344.2 & 9.7 & 35 \\
55 & 0.484 & 295.4 & 14.4 & 44 \\
60 & 0.213 & 256.8 & 47.5 & 83 \\
65 & 0.0908 & 245.0 & 0.0 & 114 \\
70 & 0.0351 & 233.8 & -5.6 & 128 \\
75 & 0.0129 & 220.9 & -11.7 & 137 \\
80 & 0.0044 & 205.4 & -14.9 & 142 \\
85 & 0.0014 & 192.6 & -12.5 & 144 \\
90 & 0.00041 & 182.2 & -10.1 & 145 \\
\hline
\end{tabular}

zontal variations of $T$ are associated with equator-to-pole differences. Thus we assume

$$
T=T(P)+\Delta T(P)\left(\cos ^{k} \lambda+C_{1}\right)
$$

where $\lambda$ is latitude and $\Delta T$ is the equator-to-pole temperature difference. $C_{1}$ is a constant dependent on $k$ such that the horizontal integral of the second term above is zero. Similarly, $F$, is assumed to be

$$
F_{s}=F_{s}(P) C_{2} \cos ^{n} \theta \quad \theta \leq \pi / 2
$$

where $\theta$ is the solar zenith angle, and $C_{2}$ is a constant dependent on $n$ such that the horizontal integral of $(8)$ is $F_{s}(P)$. In the above, $F_{s}$ is assumed to be zero for $\theta>\pi / 2$. Unfortunately, we may not assume that $F_{i}$ is independent of horizontal position due to the unexpectedly large differences shown by the SNFR net flux data on the three Pioneer Venus small probes [Suomi et al., 1979]. Thus we evaluate the infrared contribution to the integral in (1) separately for each probe. In order to provide an estimate of the globally averaged value we take the mean of the three probe integrals. Equation (1) may then be written

$$
\begin{array}{r}
0 \geq \int_{0}^{P_{0}} \frac{d F_{s}}{T}-\int_{0}^{P_{0}} \alpha \frac{\Delta T}{T^{2}} d F_{s}-\frac{1}{3} \sum_{j=1}^{3} \int_{0}^{P_{0}}\left|\frac{d F_{f}}{T}\right|_{j} \\
=I_{1}+I_{2}+I_{3}
\end{array}
$$

where $\alpha$ is a constant equal to 0.0634 for $n=k=1$ and to 0.1333 for $n=k=2$. The sum $j=1,3$ is over the three probe sites. Unless otherwise indicated, we shall use $n=k=1$ in what follows.

The integrals $I_{1}$ and $I_{2}$ in (9) are evaluated by dividing the atmosphere into layers bounded by surfaces of constant pressure. The midpoints of the layers are at altitudes of $0,5,10, \cdots$ $\mathrm{km}$ at the sounder probe location. The average temperature $\bar{T}(P)$ and the equator to pole temperature difference $\Delta T(P)$ were computed with the aid of (7) from the observed temperatures. Below $65 \mathrm{~km}$, temperatures from the sounder probe at $4.4^{\circ} \mathrm{N}$ and the north probe at $59.3^{\circ} \mathrm{N}$ were used [Seiff et al., 1979]. The probe instruments did not work below $15 \mathrm{~km}$, and the experimenters' extrapolations were used at lower altitudes.
Above $65 \mathrm{~km}$, temperatures were taken from the orbiter data [Taylor et al., 1979, this issue]. Our standard $T(P)$ and $\Delta T(P)$ profiles are listed in Table $1 a$.

The net flux $\bar{F}_{s}(P)$ is evaluated from smoothed profiles at the bounding surfaces of the layers. Differences of $\bar{F}_{s}$ across each layer define our numerical estimates of $d F_{\text {s. }}$. Adopted values are listed in Table $1 a$. The solar net flux 'global average' computed by the experimenters from sounder data [Tomasko et al., this issue $(b)]$ covers the altitude range $0-65 \mathrm{~km}$. The entry $\hat{F}_{s}=\mathbf{0}$ at the lowest altitude does not correspond to a measured flux but is included in order to account for the $15 \mathrm{~W}$ $\mathrm{m}^{-2}$ that is absorbed at the ground. The solar net flux at altitudes greater than $65 \mathrm{~km}$ was taken from a model by Tomasko et al. [this issue (a)]. The value of $145 \mathrm{~W} \mathrm{~m}^{-2}$ at the top of the atmosphere corresponds to an albedo of 0.78 for Venus [Taylor et al., this issue; Irvine et al., 1968].

The thermal net fluxes and the temperatures used to evaluate the integrals in $I_{3}$ are listed in Table $1 b$. The temperatures below $15 \mathrm{~km}$ are experimenters' extrapolations and above $65 \mathrm{~km}$ are taken from orbiter data. Direct measurements [Suomi et al., 1979; Boese et al., 1979] of $F_{i}$ cover the altitude range $15-60 \mathrm{~km}$. Since the observed net flux at the day probe includes both solar and thermal contributions, these data have been corrected by subtracting an estimated solar net radiation profile based on LSFR measurements and scattering models [Tomasko et al., this issue (a)]. Below $12.5 \mathrm{~km}$ for the north and day probes and below $27.5 \mathrm{~km}$ for the night probe, we have assumed a linear variation of $F_{i}$ with altitude due to lack of data in this region. Above $60 \mathrm{~km}$, we have assumed that over a global average the atmosphere is in radiative equilibrium. Thus we take $F_{i}=F_{s}$ for each probe.

\section{Results}

Table 2 gives values of the integrals $I_{1}, I_{2}, I_{3}$ appearing in (9). Case 1 is the standard model tabulated in Tables $1 a$ and 1b. The sum of the integrals $I_{1}+I_{2}+I_{3}$ is positive, a physical impossibility under the assumptions stated. The magnitude of the discrepancy $0.060 \mathrm{~W} \mathrm{~K}^{-1} \mathrm{~m}^{-2}$, is equivalent to a heat

\begin{tabular}{|c|c|c|c|c|c|c|}
\hline \multirow[b]{2}{*}{$Z, \mathbf{k m}$} & \multicolumn{3}{|c|}{$\boldsymbol{T}$} & \multicolumn{3}{|c|}{$F_{i}$} \\
\hline & Day & Night & North & Day & Night & North \\
\hline $\begin{array}{r}0 \\
5 \\
10 \\
15 \\
20 \\
25 \\
30 \\
35 \\
40 \\
45 \\
50 \\
55 \\
60 \\
65 \\
70 \\
75 \\
80 \\
85 \\
90\end{array}$ & $\begin{array}{l}731.0 \\
692.4 \\
652.9 \\
614.4 \\
574.3 \\
532.8 \\
490.7 \\
450.0 \\
413.4 \\
383.3 \\
346.3 \\
298.4 \\
266.8 \\
245.0 \\
233.7 \\
218.2 \\
203.6 \\
191.6 \\
181.6\end{array}$ & $\begin{array}{l}731.0 \\
692.4 \\
652.9 \\
614.4 \\
574.3 \\
532.8 \\
490.7 \\
450.1 \\
413.4 \\
383.3 \\
346.3 \\
298.4 \\
266.8 \\
245.0 \\
233.7 \\
218.1 \\
203.4 \\
191.4 \\
181.4\end{array}$ & $\begin{array}{l}735.2 \\
695.8 \\
658.1 \\
615.6 \\
573.9 \\
531.8 \\
490.3 \\
449.8 \\
411.7 \\
379.9 \\
341.6 \\
291.4 \\
243.7 \\
245.0 \\
230.0 \\
222.5 \\
212.1 \\
198.3 \\
186.3\end{array}$ & $\begin{array}{r}0 \\
13 \\
22 \\
27 \\
27 \\
24 \\
21 \\
23 \\
24 \\
28 \\
41 \\
41 \\
39 \\
83 \\
114 \\
128 \\
137 \\
142 \\
144 \\
145\end{array}$ & $\begin{array}{r}0 \\
11 \\
21 \\
32 \\
43 \\
53 \\
64 \\
68 \\
70 \\
60 \\
50 \\
64 \\
52 \\
83 \\
114 \\
128 \\
137 \\
142 \\
144 \\
145\end{array}$ & $\begin{array}{r}0 \\
17 \\
33 \\
50 \\
68 \\
80 \\
78 \\
82 \\
80 \\
85 \\
95 \\
90 \\
68 \\
83 \\
114 \\
128 \\
137 \\
142 \\
144 \\
145\end{array}$ \\
\hline
\end{tabular}
transfer of $23 \mathrm{~W} \mathrm{~m}^{-2}$ from $T=740 \mathrm{~K}$ to $T=250 \mathrm{~K}$. The mean solar heating term $I_{1}$ is large and positive. The horizontally

TABLE 1b. Profiles for the Standard Model 
varying solar heating term $I_{2}$ is very small and negative, and the infrared cooling term $I_{3}$ is also negative. The physical impossibility arises because the atmosphere cannot spontaneously transfer energy from low temperatures, where it is absorbed, to high temperatures, where it is emitted.

In case 1 above, $I_{3}$ is the mean of the infrared integrals at the three probe locations. In cases 2,3 , and $4, I_{3}$ is computed from the day, night, and north flux profiles separately. It is apparent from Table 2 that if the day probe net flux profile were typical of the planet, the integral $\int Q \mathrm{dm} / T$ would be close to zero. However, if the night and north probes were typical of the planet, the integral would be more positive than in case 1 . The variability of the net flux profiles makes it difficult to generalize. Nevertheless, we shall use case 1 as a standard model to study how $\int Q \mathrm{dm} / T$ is affected by errors of observation, assumptions about the profiles at altitudes where there are no measurements, and physical processes that might be occurring in the Venus atmosphere.

In order to assess the sensitivity of these results to the assumed parameter values we present case 5 with $n=k=2$. The horizontal variation term $I_{2}$ is still very small, and the value of $\int Q d m / T$ is still positive. In case 6 the net flux at the top of the atmosphere has been changed to $184 \mathrm{~W} \mathrm{~m}^{-2}$, corresponding to an albedo of 0.71 for Venus. This change was achieved by increasing the values of $F_{s}$ and $F$, above $65 \mathrm{~km}$ by $7,14,21,28$, $35,39 \mathrm{~W} \mathrm{~m}^{-2}$ at successively higher levels. Both $I_{1}$ and $\left|I_{3}\right|$ increase substantially, but the total integral $\int Q \mathrm{dm} / T$ stays the same.

Case 7 investigates the importance of horizontal temperature gradients. It seems surprising that the poles should be warmer than the equator at several levels in the atmosphere. However, negative $\Delta T$ in the lower atmosphere were discovered before Pioneer Venus by microwave interferometry [Sinclair et al.,. 1972; Pechmann et al., 1979], and the measurements agree with the interpretation of probe data given in Table la. Negative $\Delta T$ in the upper atmosphere were discovered by Pioneer Venus infrared radiometry. To assess the effect on the entropy integrals, all negative $\Delta T$ were set to zero in case 7 . The value of $I_{2}$ decreases slightly, but the integral $\int Q d m / T$ is still positive.

In case 8 we have replaced all the $\bar{F}_{s}$ above $65 \mathrm{~km}$ by $145 \mathrm{~W}$ $\mathrm{m}^{-2}$. This possibility exists owing to lack of probe data above that altitude. The change raises the temperature at which sunlight is absorbed, but not nearly enough to make $\int Q \mathrm{dm} / T$ negative. It is difficult to lower the temperature at which infrared radiation is emitted in the stratosphere and still have a net flux of $145 \mathrm{~W} \mathrm{~m}^{-2}$, corresponding to an effective temperature of $225 \mathrm{~K}$.

In case 9 we increase the solar net flux by the maximum amount possible due to systematic errors [Tomasko et al., this issue (b)]. This increases the solar flux by $2 \mathrm{~W} \mathrm{~m}^{-2}$ at the surface and by $25 \mathrm{~W} \mathrm{~m}^{-2}$ at $60-\mathrm{km}$ altitude, with intermediate values of the increase between these altitudes. There is no increase above $60 \mathrm{~km}$. The value of $I_{1}$ decreases significantly. Since, for consistency, we must correct the day probe data by using the higher solar flux profile, the contribution of the day probe to $I_{3}$ decreases. The contribution of the night and north probes remains the same. The integral $\int Q d m / T$ is reduced by one third, but it is still positive.

In case 10 we have raised the altitude of deep infrared emis-

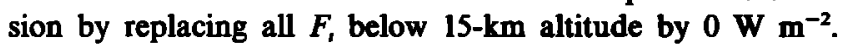
Again the effect is insignificant.

In case 11 we assume that Venus has a $18 \mathrm{~W} \mathrm{~m}^{-2}$ internal
TABLE 2. Integrals Contributing to $\int Q \mathrm{dm} / T$

\begin{tabular}{ccccc}
\hline Case & $I_{1}$ & $I_{2}$ & $I_{3}$ & $\int Q d m / T$ \\
\hline 1 & 0.512 & -0.0015 & -0.451 & 0.060 \\
2 & & & -0.504 & 0.007 \\
3 & & & -0.449 & 0.062 \\
4 & & & -0.399 & 0.112 \\
5 & 0.512 & -0.0018 & & 0.059 \\
6 & 0.695 & -0.0009 & -0.633 & 0.061 \\
7 & 0.513 & -0.0019 & -0.451 & 0.060 \\
8 & 0.498 & -0.0019 & & 0.045 \\
9 & 0.478 & -0.0007 & -0.439 & 0.038 \\
10 & & & -0.459 & 0.052 \\
11 & & & -0.514 & -0.003 \\
12 & & & -0.514 & -0.003 \\
13 & 0.452 & -0.0009 & & 0.001 \\
\hline
\end{tabular}

A blank entry indicates the case 1 value is appropriate.

heat source. We therefore change the three $F_{i}$ below $10 \mathrm{~km}$ to $18 \mathrm{~W} \mathrm{~m}^{-2}$ and add $18 \mathrm{~W} \mathrm{~m}^{-2}$ to the $F_{i}$ above $70 \mathrm{~km}$. The value of $\int Q d m / T$ is now slightly negative. An imbalance at the top of the atmosphere of $18 \mathrm{~W} \mathrm{~m}^{-2}$ is almost ruled out by orbiter data [Taylor et al., this issue], however, and is 250 times the earth's internal heat flux. In case 12 the atmosphere is assumed to be undergoing a secular cooling. To model this cooling, $13 \mathrm{~W} \mathrm{~m}^{-2}$ is added to the $F_{1}$ above $70 \mathrm{~km}$. This value gives $I_{1}+I_{2}+I_{3}$ close to zero. A net heat loss of $13 \mathrm{~W} \mathrm{~m}^{-2}$ would cool the atmosphere by about $0.5 \mathrm{~K} \mathrm{yr}^{-1}$, and could arise because of fluctuations in infrared opacity on decadal time scales. Small changes of this magnitude probably would have escaped detection during the period of modern telescopic observations of Venus.

Finally, in case 13 we assume either that the solar flux data are too low or that solar energy reaches the surface as chemically energetic rain. This hypothesis is evaluated by adding $23 \mathrm{~W} \mathrm{~m}^{-2}$ to all $\bar{F}_{s}$ between 0 and $60 \mathrm{~km}$ and adding $10 \mathrm{~W} \mathrm{~m}$ to $\bar{F}_{s}$ at $62.5 \mathrm{~km}$. The value of $\int Q \mathrm{dm} / T$ is now slightly negative.

The chemical rain hypothesis was suggested by Y. L. Yung (personal communication, 1979). At high altitudes the chemistry of the atmosphere is driven by absorption of sunlight, whereas in the lower atmosphere (below $20 \mathrm{~km}$ ) thermodynamic equilibrium chemistry should prevail, driving the system towards a state of lower free energy. Hence exchange of air between the upper and lower atmosphere results in a net deposition of heat in the lower atmosphere. The downward heat flux $F$ is approximately $q f \Delta H M / \tau$, where $q$ is the quantum yield, $f$ is the mass fraction of the absorbing constituent, $\Delta H$ is the heat of formation, $M$ is the mass per unit area of the atmospheric column, and $\tau$ is the vertical mixing time. For $f=$ $10^{-4}, q=1, \Delta H=2 \times 10^{6} \mathrm{~J} \mathrm{~kg}^{-1}, F=23 \mathrm{~W} \mathrm{~m}^{-2}$, the required mixing time is 100 days. Difficulties with this hypothesis are that the atmosphere might not mix on this rapid time scale owing to the stable stratification and that the quantum yield might be small owing to rapid recombination in the upper atmosphere.

Despite these difficulties, sulfur chemistry [Prinn, 1979; Young, 1979] provides some interesting possibilities. The reactions [Rau et al., 1973]

$$
\begin{gathered}
\mathrm{H}_{2} \mathrm{~S}+4 \mathrm{H}_{2} \mathrm{O} \rightarrow \mathrm{H}_{2} \mathrm{SO}_{4}+4 \mathrm{H}_{2} \quad \Delta H=2.3 \times 10^{6} \mathrm{~J} \mathrm{~kg}^{-1} \\
\mathrm{~S}_{8} \rightarrow 2 \mathrm{~S}_{4} \rightarrow 4 \mathrm{~S}_{2} \quad \Delta H=1.6 \times 10^{6} \mathrm{~J} \mathrm{~kg}^{-1}
\end{gathered}
$$

are driven to the right by absorption of sunlight. Recombination may be delayed if the photodissociation products precipi- 
tate out as soon as they are formed. The vapor pressures of $S_{2}$ and $S_{4}$ are in fact lower than that of $S_{8}$ [Young, 1979], and the chemical energies are greater than the energy of condensation. Thus precipitation of $S_{2}$ and $S_{4}$ accompanied by upward mixing of $S_{8}$ vapor results in a net downward energy flux. The magnitude of the flux is highly uncertain, however, and could be much lower than the $23 \mathrm{~W} \mathrm{~m}^{-2}$ required to explain the Pioneer Venus observations.

Mechanical transport of energy downward has not been treated as a separate case in Table 2. Since there is no external source of mechanical energy, any work done by the upper atmosphere on the lower atmosphere must come from thermal sources, and therefore must satisfy (1). But since we cannot satisfy (1) without postulating additional heat sources (internal heat, secular cooling of the atmosphere, chemically energetic rain, etc.), we conclude that downward mechanical energy transport is quantatively incapable of explaining the net flux data when the probe locations are taken to be typical.

In summary, we cannot claim to understand the high surface temperatures of Venus on the basis of the Pioneer Venus observations. If the variability of the observed infrared net flux profiles is real and if the night and north profiles are not anomalously high due to local conditions, then a transfer of $23 \mathrm{~W} \mathrm{~m}^{-2}$ from the upper to the lower atmosphere is implied. This transfer cannot be due to any steady state, thermally driven process in the atmosphere. We conclude either that some of the net flux data are in error or that they are from anomalous regions or else that there is another source of energy for the deep atmosphere. Examples include $23 \mathrm{~W} \mathrm{~m}^{-2}$ internal heat source, a secular cooling of the atmosphere, or a chemically energetic rain carrying solar energy downward.

Acknowledgment. Contribution 3368 of the Division of Geological and Planetary Sciences, California Institute of Technology.

\section{REFERENCES}

Boese, R. W., J. B. Pollack, and P. M. Silvaggio, First results from the large probe infrared radiometer experiment, Science, 203, 797, 1979.

Goody, R., and A. Robinson, A discussion of the deep circulation of the atmosphere of Venus, Astrophys. J., 146, 339, 1966.

Irvine, W. M., Monochromatic phase curves and albedos for Venus, $J$. Atmos. Sci., 25, 610, 1968.

Pechmann, J. B., D. O. Muhleman, and G. L. Berge, Polar heating and the shape of Venus, Nature, 279, 618, 1979.
Prinn, R. G., On the possible roles of gaseous sulfur and sulfanes in the atmosphere of Venus, Geophys. Res. Lett., 6, 807, 1979.

Rau, H., T. R. N. Kutty, and J. R. F. Guedes de Carvalho, Thermodynamics of sulfur vapour, J. Chem. Thermodyn., 5, 833, 1973.

Sagan, C., The surface temperature of Venus, Astron. J., 65, 352, 1960.

Schubert, G., C. C. Counselman, J. Hansen, S. S. Limaye, G. Pettengill, A. Seiff, I. I. Shapiro, V. E. Suomi, F. Taylor, L. Travis, R. Woo, and R. E. Young, Dynamics, winds, circulation, and turbulence in the atmosphere of Venus, Space Sci. Rev., 20, 357, 1977.

Schubert, G., et al., Structure and circulation of the Venus atmosphere, J. Geophys. Res., this issue.

Seiff, A., D. B. Kirk, R. E. Young, S. C. Sommer, R. C. Blanchard, J. $T$. Findlay, and G. M. Kelly, Thermal contrast in the atmosphere of Venus: Initial appraisal from Pioneer Venus probe data, Science, 205, 46, 1979.

Seiff, A., et al., Thermal structure, thermal constraints, and dynamical observations in the atmosphere of Venus: Results of in situ measurements from the four Pioneer Venus probes, J. Geophys. Res., this issue.

Sinclair, A. C. E., J. P. Basart, D. Buhl, and W. A. Gale, Precision interferometer observations of Venus at 11.1-centimeter wavelength, Astrophys. J., 175, 555, 1972.

Suomi, V. E., L. A. Sromovsky, and H. E. Revercomb, Preliminary results of the Pioneer Venus small probe net flux radiometer experiment, Science, 205, 82, 1979.

Suomi, V. E., L. A. Sromovsky, and H. E. Revercomb, Net radiation in the atmosphere of Venus: Measurements and interpretation, $J$. Geophys. Res., this issue.

Taylor, F. W., D. J. Diner, L. S. Elson, D. J. McCleese, J. V. Martonchik, J. Delderfield, S. P. Bradley, J. T. Schofield, J. C. Gille, and M. T. Coffey, Temperature, cloud structure, and dynamics of Venus middle atmosphere by infrared remote sensing from Pioneer orbiter, Science, 205, 65, 1979.

Taylor, F. W., et al., Structure and meteorology of the middle atmosphere of Venus: Infrared remote sensing from the Pioneer orbiter, J. Geophys. Res., this issue.

Tomasko, M. G., R. Boese, A. P. Ingersoll, A. A. Lacis, S. S. Limaye, J. B. Pollack, A. Seiff, A. I. Stewart, V. E. Suomi, and F. W. Taylor, The thermal balance of the atmosphere of Venus, Space Sci. Rev., 20, 389, 1977.

Tomasko, M. G., L. R. Doose, and P. H. Smith, Absorption of sunlight in the atmosphere of Venus, Science, 205, 80, 1979.

Tomasko, M. G., et al., Thermal balance of Venus in light of the Pioneer Venus mission, J. Geophys. Res., this issue.

Tomasko, M. G., L. R. Doose, P. H. Smith, and A. P. Odell, Measurements of the flux of sunlight in the atmosphere of Venus, $J$. Geophys. Res., this issue.

Young, A. T., Chemistry and thermodynamics of sulfur on Venus, Geophys. Res. Lett., 6, 1979.

(Received November 18, 1979;

revised May 8, 1980;

accepted May 9, 1980.) 